\title{
Simulated driving in obstructive sleep apnoea-hypopnoea; effects of oral appliances and continuous positive airway pressure
}

\author{
Aarnoud Hoekema • Boudewijn Stegenga • \\ Marije Bakker • Wiebo H. Brouwer • \\ Lambert G. M. de Bont • Peter J. Wijkstra • \\ Johannes H. van der Hoeven
}

Published online: 24 January 2007

(C) Springer-Verlag 2007

\begin{abstract}
Impaired simulated driving performance has been demonstrated in obstructive sleep apnoea-hypopnoea syndrome (OSAHS) patients. Although continuous positive airway pressure (CPAP) generally improves simulated driving performance, the effects of oral-appliance (OA) therapy are unknown. The aims of this study were to determine to what extent OSAHS patients have more difficulty with a monotonous simulated driving test when compared with control subjects and to compare the effects of OA with CPAP therapy. Simulated driving performance was evaluated in 20 OSAHS patients and 16 control subjects during a 25-min driving test. After randomization,
\end{abstract}

\footnotetext{
A. Hoekema $(\bowtie) \cdot$ B. Stegenga $\cdot$ L. G. M. de Bont Department of Oral and Maxillofacial Surgery, University Medical Center Groningen, University of Groningen, Hanzeplein 1,

P.O. Box 30.001, 9700 RB Groningen, The Netherlands

e-mail: a.hoekema@kchir.umcg.nl
}

M. Bakker · W. H. Brouwer

Department of Clinical Neuropsychology,

University Medical Center Groningen, University of Groningen, Hanzeplein 1,

P.O. Box 30.001, 9700 RB Groningen, The Netherlands

\section{P. J. Wijkstra}

Department of Home Mechanical Ventilation/Pulmonary

Diseases, University Medical Center Groningen,

University of Groningen,

Hanzeplein 1,

P.O. Box 30.001, 9700 RB Groningen, The Netherlands

J. H. van der Hoeven

Department of Clinical Neurophysiology,

University Medical Center Groningen, University of Groningen,

Hanzeplein 1,

P.O. Box 30.001, 9700 RB Groningen, The Netherlands ten patients started OA and CPAP therapy, respectively. After 2 to 3 months of treatment, patients repeated the driving test. At baseline, the total number of lapses of attention during driving was significantly higher in OSAHS patients as compared with control subjects. As a result of treatment, the total number of lapses of attention was significantly decreased in both the OA and CPAP group. When comparing driving performance between the OA and CPAP group, no significant differences were noted. OSAHS patients perform worse on a simulated driving test when compared with control subjects. When evaluating the effects of treatment, adequate OSAHS management with either OA or CPAP therapy usually resulted in substantial improvements of simulated driving. Conclusions beyond both treatments improving simulated driving performance are, however, not justified by the data in the present study.

Keywords Sleep apnoea syndromes · Automobile driving · Orthodontic appliances $\cdot$ Positive-pressure ventilation

\section{Introduction}

The obstructive sleep apnoea-hypopnoea syndrome (OSAHS) is a common sleep-related breathing disorder associated with serious neurocognitive and cardiovascular sequelae [1]. In OSAHS patients, respiratory-related arousals during sleep cause sleep fragmentation and a depletion of rapid eye movement and slow-wave sleep, ultimately resulting in excessive daytime sleepiness. Daytime sleepiness may adversely affect daytime performance and consequently, among other sequelae, influence driving performance [2, 3]. Studies evaluating subjective (self- 
reports) or objective (motor vehicle department) records sustain the opinion that OSAHS confers an increased risk for accidents when driving [4, 5]. In addition, performance on driving simulator and vigilance tests of varying complexity have been shown impaired in OSAHS patients [6-10]. Driving is a multifaceted and multilevelled skill, and it is impossible to assess all of its aspects in a simulator [11]. Depending on the nature of the disorder studied, key driving tasks can be selected for clinical assessment. In OSAHS patients, the primary problem is to sustain attention in monotonous situations. Therefore, the most relevant driving task is prolonged driving on a monotonous highway. Most simulator studies in OSAHS patients use a variation of this task [6-10].

When evaluating the effects of OSAHS treatment on motor vehicle crash risk, marked improvements are generally reported. Studies evaluating subjective and objective records indicate that continuous positive airway pressure (CPAP) therapy reduces collision and crash frequency in OSAHS patients $[4,8,12]$. In addition, successful treatment with CPAP therapy generally results in improvements of simulated driving performance [13-15]. Similar improvements in simulated driving performance have been demonstrated after successful OSAHS management by means of uvulopalatopharyngoplasty [6]. Over the past decade, oral-appliance (OA) therapy has emerged as a well-proven alternative in the treatment of OSAHS patients that is accepted by the sleep society $[16,17]$. An OA aims at relieving upper airway collapse during sleep by modifying the position of the mandible, the tongue, and pharyngeal structures. However, the effects of OA therapy on simulated driving performance have not been evaluated to date [16].

The aims of this study were to determine to what extent OSAHS patients have more difficulty with a monotonous simulated driving test when compared with control subjects and to compare the effects of OA with CPAP therapy.

\section{Materials and methods}

\section{Patients}

Control subjects and a subset of OSAHS patients, recruited for a randomized trial comparing the effectiveness of OA and CPAP therapy, comprised the study population. All subjects were required to be aged between 21 and 70 years, in the possession of a drivers licence, not involved in shiftwork or night-time work, and otherwise fit to drive.

Thirty consecutive patients who underwent polysomnography (baseline polysomnography) and were diagnosed as having OSAHS with an apnoea-hypopnoea index $(\mathrm{AHI})>5$ were considered for inclusion in the present study. Patients were recruited through the Department of Home Mechanical Ventilation of the University Medical Center Groningen (Groningen, The Netherlands) in the period March to June 2004. Patients were excluded in case of previous OSAHS treatment, clearly reversible morphological airway abnormalities, endocrine dysfunction (one patient with hypothyroidism, two patients with pituitary adenoma), a reported or documented history of severe cardiac or pulmonary disease (one patient with daytime respiratory insufficiency), moderate or severe periodic limb movement disorder (i.e. periodic limb movement index $>25$ ), or a psychological condition precluding informed consent. Patients with a dental status that could complicate OA therapy were also excluded (three patients had an insufficient number of teeth, one patient had extensive periodontal disease) [16]. Because two of the remaining 22 patients refused participation, 20 OSAHS patients could eventually be enrolled. Sixteen healthy subjects matched for age and without signs of a neurological or psychiatric disorder were recruited as control group. The control group comprised a diverse compilation of hospital employees who had no knowledge or clinical suspicion by questionnaire analyses of having a sleeping disorder. The randomized trial was approved by the Groningen University Medical Center's ethics committee (METc 2002/032). Written informed consent was obtained from each patient before enrolment after full explanation of the procedure. In addition, all control subjects consented to participation in the study after full explanation of the procedure.

\section{Study design}

Of the 20 OSAHS patients, ten were allocated to OA therapy and ten to CPAP therapy by means of block randomization [18]. OA therapy was initiated in the Department of Oral and Maxillofacial Surgery and was supervised by one dentist. CPAP therapy was initiated in the Department of Home Mechanical Ventilation and was supervised by one pulmologist. It was not possible to blind the patients or clinicians to treatment assignment. At baseline all OSAHS patients and control subjects completed the Epworth sleepiness scale [19] and an in-house questionnaire evaluating medication prescription, caffeine consumption, tobacco use, and driving experience. Thereupon, simulated driving performance was evaluated in both groups during a 25 -min driving test.

The OA used in this study (Thornton Adjustable Positioner, Airway Management, Dallas, TX, USA) consisted of two separate parts and positioned the patient's mandible in a forwards and downwards position [20]. The upper part was supported by the dentition of the maxilla and the lower part by the dentition of the mandible. By turning a propulsion screw incorporated anteriorly in the 
appliance, the patient could adjust the amount of mandibular advancement in $0.2-\mathrm{mm}$ increments. The maximum advancement of the mandible was determined with a George-Gauge $^{\text {TM }}$ (H-Orthodontics, Michigan City, IN, USA) before OA therapy began. Initially, the mandible was set at approximately $50 \%$ of the patient's maximum advancement. After patients became accustomed to the OA during a 2-week period, they returned for a check-up visit. They were instructed to adjust the OA over the following 6 weeks. To do so, patients were instructed to advance the mandible each night with one to two increments (i.e. 0.2 to $0.4 \mathrm{~mm}$ ) whenever OSAHS-symptomatology persisted (e.g. snoring, apnoeas, hypopnoeas, or excessive sleepiness). This titration of the appliance was continued until symptoms abated or until further advancement of the mandible caused discomfort.

CPAP titration was performed during an afternoon nap. This technique, aimed at abolishing all signs of apnoea, hypopnoea, and snoring, has been shown an appropriate procedure for the effective titration of CPAP [21]. After titration, an 8-week follow-up period was arranged that allowed for habituation and, if necessary, adjustments of CPAP therapy.

After patients used an OA or CPAP for 8 weeks, the treatment effect was assessed with a second polysomnographic study. For patients whose AHI was still $\geq 5$, treatment was adjusted, if possible, to improve effectiveness. For this purpose OA-treated patients were instructed to protrude their mandible with the appliance to the maximum point of protrusion, which was still comfortable during a full therapeutic night. In CPAP-treated patients, the pressure was raised by the pulmologist supervising therapy with 1 or $2 \mathrm{~cm} \mathrm{H}_{2} \mathrm{O}$ (depending on the severity of residual OSAHS with CPAP). In these patients, the follow-up period was extended with another 4 weeks. The effect was then assessed with a third polysomnographic study. This adjustment sequence was continued until the AHI was $<5$ or until the adjustments became uncomfortable for the patient. Follow-up review ended with a patient's final polysomnographic evaluation or when a patient discontinued treatment because of poor tolerance or another reason. At their final follow-up review, patients again completed the Epworth sleepiness scale and driving test performed at baseline. In addition, treatment usage was evaluated at this stage by asking patients how many nights per week and how many hours per night they used their treatment.

Treatment was considered effective when the AHI either was $<5$ or showed "substantial reduction" [16], defined as reduction in the index of at least $50 \%$ from the baseline value to a value of $<20$ in a patient who had no symptoms while using therapy. Patients not meeting these criteria at their final review were considered "nonresponsive" to treatment.
Polysomnography

Polysomnography (Embla ${ }^{\circledR}$ A10 digital recorder, Medcare, Reykjavik, Iceland) for baseline and follow-up evaluations was conducted ambulatory in the patient's home situation. Each study started 11 A.M. and stopped 9 A.M. the next morning. Surface electroencephalography, submental electromyography, and left and right electrooculography were used to stage sleep. A pulsoximeter (Oximeter Flex Sensor$8000 \mathrm{~J}-3$, Medcare) was used to record oxyhaemoglobin saturation. Electrocardiography was used to monitor cardiac function. Oronasal airflow was recorded with a pressure cannula. Respiratory effort was monitored with thoracic and abdominal strain gauges. An anterior tibial electromyogram was recorded to screen for periodic limb movements. Outcomes were limited to the time in bed part of the study. Standardized criteria were used to score apnoeas and hypopnoeas [22], arousals [23], sleep stages [24], and periodic limb movements [25]. All polysomnographic studies were evaluated and scored by one neurophysiologist (J.H.) who was unaware of the patient's treatment assignment.

\section{Driving simulator}

The driving test was performed on the driving simulator of the Department of Neuropsychology [26, 27]. All subjects were tested between noon and 2 P.M. In OSAHS patients, the second driving test was conducted at the same point of time as the first evaluation. Instructions were to refrain from stimulating products (e.g. caffeine) $3 \mathrm{~h}$ before testing and not to smoke half an hour before testing. To keep testing conditions as consistent as possible lights were dimmed, noise was shut out, and room temperature was kept at $22^{\circ} \mathrm{C}$. The driving test had to be completed in absence of company, including the test leader (M.B.). Subjects were seated in a comfortable office chair behind a 21 -in. computer screen on which the scene of a straight road was projected. Using a computer game steering wheel, the lateral position on this road could be controlled. In all conditions, the speed of driving was fixed at $50 \mathrm{~km} / \mathrm{h}$, and the only objective was to drive as straight as possible in the middle of the right lane, while compensating for the effect of a low amplitude unpredictable "side-wind" signal (superposition of $1 / 15,1 / 7.5$, and $1 / 3.75 \mathrm{~Hz}$ sinusoids). Because of this unpredictable "side wind", subjects had to stay alert continuously to accomplish the task without getting out of lane. After a 5-min practice, it was ascertained that subjects had mastered the task. Subsequently, all subjects performed a 25 -min driving test in a monotonous situation. Side-wind amplitude was set low so that all subjects could easily perform the task in the first $5 \mathrm{~min}$, showing standard deviations of lateral position 
comparable to straight road driving in "the real world" [28].

During the test, every $15 \mathrm{~s}$, the standard deviation of the lateral position (SDLP) was calculated. Driving performance was expressed in two separate parameters:

- The number of lapses of attention (LOA). For this purpose, the median of 20 consecutive SDLP's was computed, resulting in one number every $5 \mathrm{~min}$. Subjects were considered to perform at a normal level during the first five test minutes, unaffected yet by the monotonous task. A lapse of attention was defined as a SDLP in a given 15 -s period that was twice as high as the median SDLP of the first five test minutes.

- The time course SDLP to assess a possible individual increase in the five median SDLP's. For this purpose, an individual linear regression analysis was computed of the five consecutive median SDLP's, resulting in a slope coefficient. The higher this number, the steeper the regression line and the larger the adverse effect of time on simulated driving performance.

\section{Analysis}

Statistical analyses were performed using the Statistical Package for the Social Sciences version 12.0 (SPSS, Chicago, IL, USA). Means and standard deviations or medians and interquartile ranges in skewed distributions are reported. The total number of LOA during the driving test was used as primary outcome measure. Other driving test outcomes included the number of LOA per 5-min time stage (five epochs) and the slope coefficient of time course SDLP. To compare outcomes between the groups, independent sample $t$-tests were used (Mann-Whitney $U$ tests for variables with skewed distributions). Differences between baseline and follow-up variables in the OA and CPAP groups were compared with paired Student's $t$-tests (Wilcoxon's signed ranks tests for variables with skewed distributions). To measure correlations in the OSASHS group between the total number of LOA, AHI, and Epworth sleepiness scale, Spearman's rank correlation coefficients were calculated. A significance level of $p<0.05$ was predefined in all cases.

\section{Results}

OSAHS patients vs control subjects

When comparing the 20 OSAHS patients and the 16 control subjects, no significant differences in age, daily caffeine and nicotine consumption, or driving experience were observed at baseline (Table 1). With respect to the Epworth sleepiness scale, a significantly higher level of subjective sleepiness was observed in the OSAHS patient group.

Before treatment, driving performance, as indicated by the total number of LOA, was worse in OSAHS patients than in control subjects (Table 2, Fig. 1). In addition, there were significantly more LOA's in the second to the fifth epoch in OSAHS patients than in the control subjects. The slope coefficient of the time course of SDLP significantly differed in favour of the control subjects. In the OSAHS patients, no significant correlation was observed between the total number of LOA and the AHI or the Epworth sleepiness scale.

\section{Effects of OA and CPAP therapy}

Of the ten patients that were allocated to OA therapy, one patient's symptoms and driving performance deteriorated (increase in the total number of LOA from 17 to 26) due to a severe periodic limb movement syndrome at final followup review (increase in periodic limb movement index from 0 to 62). This deterioration after treatment could not be explained by a residual OSAHS (reduction AHI from 28 to 0 ) nor were there any other factors that could explain this phenomenon (e.g. anthropometry or driving experience). This patient was, therefore, excluded from further analysis. Baseline characteristics were similar for the nine OA- and ten CPAP-treated patients (Table 3). At final review, the mean advancement of the mandible with the OA was $89 \pm$ $23 \%$ of the maximum advancement. The mean CPAP pressure was $8.7 \pm 2.2 \mathrm{~cm} \mathrm{H}_{2} \mathrm{O}$ at final review. The median period to final review was 81 (interquartile range 72-93) days in the OA group and 79 (interquartile range 63-102) days in the CPAP group $(p>0.05)$.

Treatment was effective for eight patients in the OA group and for nine patients in the CPAP group. The two remaining patients were "nonresponsive" to treatment. As a result of OA and CPAP therapy, the AHI and $\mathrm{MinSaO}_{2}$ improved significantly in both groups (Table 3). No significant differences in the $\mathrm{AHI}$ and $\mathrm{MinSaO}_{2}$ were found between the treatment groups at final follow-up review. The Epworth sleepiness scale was significantly improved only in the CPAP-treated group. When comparing Epworth sleepiness scale values at final review, no significant differences were noted between the two groups. There were no significant differences between the treatment modalities with regard to usage (Table 3).

No significant differences in driving performance were observed between the two groups at baseline (Table 4). After therapy, the total number of LOA was significantly decreased in both groups. In one OA- and one CPAPtreated patient, the total number of LOA was increased at final follow-up review (Fig. 1). Both patients were "nonresponsive" to treatment. Significant improvements in 
Table 1 Baseline characteristics for OSAHS patients and control subjects

\begin{tabular}{llll}
\hline Variable & OSAHS patients $(n=20)^{\mathrm{a}}$ & ${\text { Control subjects }(n=16)^{\mathrm{a}}}^{\text {Difference }^{\mathrm{b}}}$ \\
\hline Male/female ratio & $17 / 3$ & $13 / 3$ & - \\
Age (year) & $48.7 \pm 11.2$ & $48.7 \pm 10.0$ & $\mathrm{NS}$ \\
$\mathrm{BMI}\left(\mathrm{kg} / \mathrm{m}^{2}\right)$ & $33.2 \pm 5.7$ & - & - \\
Neck circumference $(\mathrm{cm})$ & $44.2 \pm 2.7$ & - & - \\
Epworth sleepiness scale & $13.5 \pm 6.5$ & $6.1 \pm 4.3$ & $p=0.000$ \\
Caffeine (units/day) & $5.5 \pm 3.3$ & $6.7 \pm 3.4$ & $\mathrm{NS}$ \\
Nicotine (units/day) & $0.0(0.0-9.5)$ & $0.5(0.0-12.5)$ & $\mathrm{NS}$ \\
Driving experience & & & $\mathrm{NS}$ \\
Years of driving experience & $29 \pm 10$ & $29 \pm 11$ & $\mathrm{NS}$ \\
Annual number of kilometres $\times 10^{3}$ & $15(10-32)$ & - & - \\
AHI (events/hour) & $49.1 \pm 33.3$ & - & - \\
$\mathrm{MinSaO}_{2}(\%)$ & $76.4 \pm 9.7$ & & \\
\hline
\end{tabular}

AHI apnoea-hypopnoea index; $B M I$ body mass index; $N S$ not significant; $\mathrm{MinSaO}_{2}$ lowest oxyhaemoglobin saturation during sleep; $O S A H S$ obstructive sleep apnoea-hypopnoea syndrome

${ }^{a}$ Plus-minus values are means \pm standard deviations, values with additives in parenthesis are medians with interquartile ranges.

${ }^{\mathrm{b}}$ Significance for the difference in baseline characteristics between the OSAHS patients and control subjects.

the remaining driving test outcomes were observed only in the CPAP group for the number of LOA in the fifth epoch. No significant differences were noted between the driving test data at final review for OA and CPAP therapy. For the OSAHS patient group, a significant correlation was demonstrated between changes in the total number of LOA and the Epworth sleepiness scale after treatment $(r=0.570, p=0.01)$.

\section{Discussion}

This study shows that OSAHS patients clearly have difficulty with a simulated driving test when compared with control subjects. During the 25-min driving test, OSAHS patients showed a considerable deterioration in simulated driving performance. When evaluating the effects of OA and CPAP treatment on driving performance, significant improvements in the total number of lapses of attention were observed in both treatment groups. These findings suggest that effective OSAHS treatment, with either OA or CPAP therapy, contributes to an improvement of simulated driving performance.

Based on the total number of LOA, OSAHS patients show worse driving performance when compared with control subjects. These findings are in keeping with other studies that also showed impaired performance in OSAHS patients during various tests of simulated driving $[7,9,10]$. The present study also suggests that simulated driving performance in OSAHS patients deteriorates with time on task, as indicated by the slope coefficient of the time course SDLP. Because already in the second epoch significantly more LOA occurred in the OSAHS group, a decline in driving performance may be expected after a relatively short period of time (i.e. $10 \mathrm{~min}$ ). In contrast to several other simulator studies [2], the present study only evaluated

Table 2 Baseline driving test outcomes for OSAHS patients and control subjects

\begin{tabular}{lccc}
\hline Variable & OSAHS patients $(n=20)^{\mathrm{a}}$ & ${\text { Control subjects }(n=16)^{\mathrm{a}}}$ & \\
\hline Number of LOA & & $0.0(0.0-1.8)$ & $p=0.000$ \\
Total $(0-25 \mathrm{~min})$ & $10.0(1.3-14.5)$ & $0.0(0.0-0.0)$ & NS \\
First epoch $(0-5 \mathrm{~min})$ & $0.0(0.0-0.0)$ & $0.0(0.0-0.0)$ & $p=0.021$ \\
Second epoch $(6-10 \mathrm{~min})$ & $0.0(0.0-1.0)$ & $0.0(0.0-0.0)$ & $p=0.005$ \\
Third epoch $(11-15 \mathrm{~min})$ & $1.0(0.0-4.8)$ & $0.0(0.0-0.0)$ & $p=0.000$ \\
Fourth epoch $(16-20 \mathrm{~min})$ & $3.0(0.3-5.8)$ & $0.0(0.0-0.0)$ & $p=0.001$ \\
Fifth epoch $(21-25 \mathrm{~min})$ & $2.5(0.0-6.0)$ & $0.03(-0.15-0.17)$ & $p=0.006$ \\
Slope coefficient of time course SDLP & $0.41(0.06-0.70)$ & \\
\hline
\end{tabular}

$L O A$ lapses of attention; $N S$ not significant; OSAHS obstructive sleep apnoea-hypopnoea syndrome; $S D L P$ standard deviation of the lateral position

${ }^{\text {a }}$ Values with additives in parenthesis are medians with interquartile ranges.

${ }^{\mathrm{b}}$ Significance for the difference in baseline driving test outcomes between the OSAHS patients and control subjects. 
Fig. 1 Individual values of the total number of LOA in OSAHS patients and control subjects. In OSAHS patients, the total number of LOA at baseline and final follow-up review are depicted according to treatment group (i.e. $O A$ or $C P A P$ ). The total number of LOA are not depicted for one patient allocated to $\mathrm{OA}$ therapy. This patient was excluded from follow-up analysis because his symptoms and driving performance deteriorated due to a severe periodic limb movement syndrome at the final follow-up review (increase in periodic limb movement index from 0 to 62). At baseline, the total number of LOA for this patient was 17

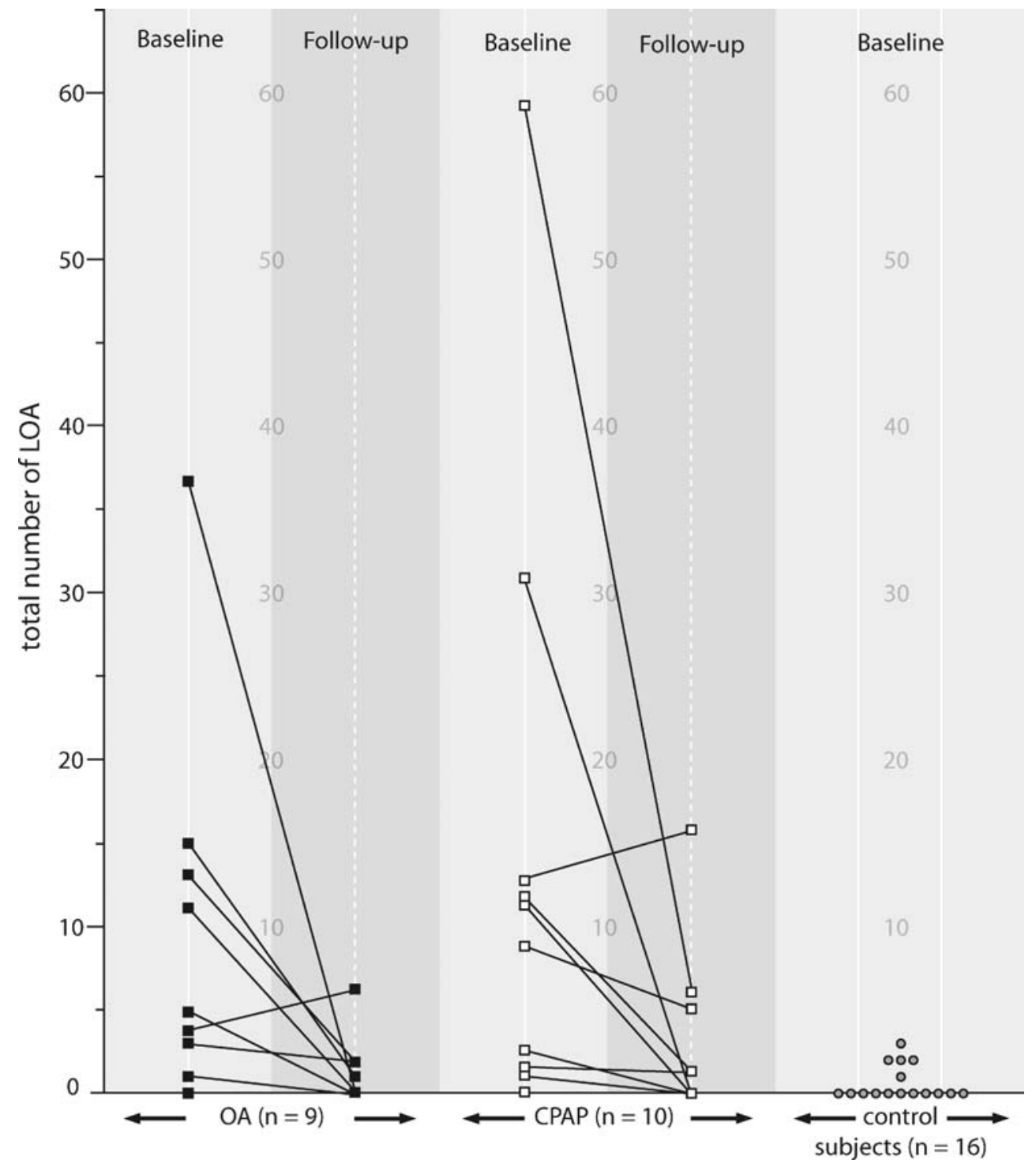

primary vehicle control. Secondary tasks, such as reaction time to specific stimuli, have been shown to actually stimulate drivers when performing a simulator test [29]. Because the driving test used in the present study proved to be sensitive to distinguish OSAHS patients from controls, it appears a valid measure of the fatiguing effects of a monotonous task.

Despite the fact that OSAHS patients performed worse as a group, not all patients showed impaired simulator performance when compared with the control subjects. This variability in simulated driving performance has also been observed in previous studies $[7,9,13]$. In the present study, this phenomenon may be explained by inclusion of OSAHS patients of varying severity. However, at baseline, significant correlations between the total number of LOA and the AHI or Epworth sleepiness scale could not be demonstrated. These findings are corroborated by other studies that also fail to demonstrate strong relationships between these parameters and driving simulator performance [7, 10]. Therefore, the question remains as to what extent other factors, besides OSAHS severity and symptoms, affect sustained driving performance in untreated patients (e.g. personality or coping strategies).

Especially in the case of a more deviant performance at baseline, adequate OSAHS management by means of either OA or CPAP therapy usually resulted in substantial improvements of simulated driving. The correlation observed between changes in the total number of LOA and Epworth sleepiness scale values suggests that adequate OSAHS management ameliorates driving performance. This suggestion is supported by the fact that worse driving performance at the final review was observed in those patients who were "nonresponsive" to OA or CPAP therapy. Other studies evaluating simulator performance after CPAP therapy or uvulopalatopharyngoplasty usually also observe improvements in driving performance [6, 13- 
Table 3 Baseline and follow-up characteristics for OA- and CPAP-treated patients

\begin{tabular}{|c|c|c|c|}
\hline Variable & $\mathrm{OA}(n=9)^{\mathrm{a}}$ & $\operatorname{CPAP}(n=10)^{\mathrm{a}}$ & Difference $^{\mathrm{b}}$ \\
\hline Male/female ratio & $7 / 2$ & $9 / 1$ & - \\
\hline Age (year) & $47.6 \pm 11.0$ & $49.7 \pm 12.4$ & NS \\
\hline \multicolumn{4}{|l|}{ BMI $\left(\mathrm{kg} / \mathrm{m}^{2}\right)$} \\
\hline Baseline & $32.5 \pm 6.6$ & $34.2 \pm 5.2$ & NS \\
\hline \multicolumn{4}{|l|}{ Neck circumference $(\mathrm{cm})$} \\
\hline Baseline & $43.3 \pm 3.1$ & $45.0 \pm 2.4$ & NS \\
\hline \multicolumn{4}{|l|}{ Epworth sleepiness scale } \\
\hline Baseline & $11.3 \pm 7.9$ & $15.0 \pm 5.0$ & NS \\
\hline Follow-up review ${ }^{\mathrm{c}}$ & $6.7 \pm 6.0$ & $5.5 \pm 4.1$ & NS \\
\hline$\Delta$ & $-4.7 \pm 7.7$ & $-9.5 \pm 6.6^{* * * * *}$ & NS \\
\hline \multicolumn{4}{|l|}{ Caffeine (units/day) } \\
\hline Baseline & $6.2 \pm 3.6$ & $5.1 \pm 3.2$ & NS \\
\hline \multicolumn{4}{|l|}{ Nicotine (units/day) } \\
\hline Baseline & $0.0(0.0-7.0)$ & $0.0(0.0-8.5)$ & NS \\
\hline \multicolumn{4}{|l|}{ Driving experience } \\
\hline Years of driving experience & $28 \pm 9$ & $30 \pm 12$ & NS \\
\hline Annual number of kilometres $\times 10^{3}$ & $15(5-23)$ & $18(10-50)$ & NS \\
\hline \multicolumn{4}{|l|}{ AHI (events/hour) } \\
\hline Baseline & $47.7(12.0-92.6)$ & $54.6(22.8-65.1)$ & NS \\
\hline Follow-up review ${ }^{\mathrm{c}}$ & $5.3(0.2-9.9)$ & $0.0(0.0-4.8)$ & NS \\
\hline$\Delta$ & $-42.5(-68.8 \text { to }-12.0)^{* *}$ & $-54.6(-64.9$ to -16.2$) * * * *$ & NS \\
\hline \multicolumn{4}{|l|}{$\mathrm{Min} \mathrm{SaO}_{2}(\%)$} \\
\hline Baseline & $78.0(74.0-80.5)$ & $79.5(66.8-83.3)$ & NS \\
\hline Follow-up review ${ }^{\mathrm{c}}$ & $91.0(82.0-92.5)$ & $92.0(88.0-94.0)$ & NS \\
\hline$\Delta$ & $9.0(6.0-15.0)^{*}$ & $12.5(9.5-24.3)^{* * *}$ & NS \\
\hline \multicolumn{4}{|l|}{ Treatment usage } \\
\hline Nights per week & $6.8 \pm 0.4$ & $6.8 \pm 0.6$ & NS \\
\hline Hours per night & $7.0 \pm 0.9$ & $6.7 \pm 1.4$ & NS \\
\hline
\end{tabular}

AHI apnoea-hypopnoea index; $B M I$ body mass index; $C P A P$ continuous positive airway pressure; $\mathrm{NS}_{\text {not }}$ significant; $\mathrm{MinSaO}$ lowest oxyhemoglobin saturation during sleep; $O A$ oral appliance

${ }^{a}$ Plus-minus values are means \pm standard deviations, values with additives in parenthesis are medians with interquartile ranges.

${ }^{\mathrm{b}}$ Significance for the difference in baseline, follow-up, or $\Delta$ outcomes between treatment groups.

${ }^{\mathrm{c}}$ The median treatment period from baseline until final follow-up review was 81 (interquartile range $72-93$ ) days in the OA group and 79 (interquartile range 63-102) days in the CPAP group $(p>0.05)$.

${ }^{*} p=0.01$ for difference between baseline and follow-up outcomes within treatment group

$* * p=0.008$ for difference between baseline and follow-up outcomes within treatment group

$* * * p=0.007$ for difference between baseline and follow-up outcomes within treatment group

$* * * * p=0.005$ for difference between baseline and follow-up outcomes within treatment group

$* * * * * p=0.001$ for difference between baseline and follow-up outcomes within treatment group

15, 30]. To our knowledge, the present study is the first to study the effects of OA therapy on simulated driving performance. Although both groups showed significant improvements in the total number of LOA, significant improvements for the number of LOA in the fifth epoch were observed only for the subjects treated with CPAP. These findings may suggest a more pronounced effect of CPAP therapy on simulated driving performance. However, it is more likely that methodological issues explain this phenomenon. First, our study was of explorative character and not based on a power calculation. Second, despite nonsignificant differences, baseline values indicated more severe OSAHS and worse simulator performance in the CPAP group. In addition, with respect to baseline and follow-up values, no significant differences in simulated driving performance could be demonstrated between CPAP- and OA-treated patients.

Despite the observed differences with control subjects and the favourable effects of treatment on simulator performance, there are a number of limitations to the present study. The first limitation refers to the control group. Control subjects were hospital employees and approximately $70 \%$ agreed after being asked to participate. Therefore, selection bias may have occurred. In addition, because polysomnographic data were not available in this group, subjects with "sub-clinical" OSAHS may have been included. However, questionnaire analyses raised no evidence of OSAHS symptoms in the control subjects. 
Table 4 Baseline and follow-up driving test outcomes for OA- and CPAP-treated patients

\begin{tabular}{|c|c|c|c|}
\hline Variable & OA $(n=9)^{\mathrm{a}}$ & $\operatorname{CPAP}(n=10)^{\mathrm{a}}$ & Difference $^{b}$ \\
\hline \multicolumn{4}{|l|}{ Number of LOA } \\
\hline \multicolumn{4}{|l|}{ Total (0-25 min) } \\
\hline Baseline & $5.0(2.0-14.0)$ & $10.0(1.0-16.8)$ & NS \\
\hline Follow-up review ${ }^{\mathrm{c}}$ & $0.0(0.0-2.0)$ & $0.5(0.0-5.3)$ & NS \\
\hline$\Delta$ & $-5.0(-12.5 \text { to }-0.5)^{* *}$ & $-3.0(-16.0-0.0)^{* *}$ & NS \\
\hline \multicolumn{4}{|l|}{ First epoch (0-5 min) } \\
\hline Baseline & $0.0(0.0-1.0)$ & $0.0(0.0-0.0)$ & NS \\
\hline Follow-up review ${ }^{c}$ & $0.0(0.0-0.5)$ & $0.0(0.0-0.0)$ & NS \\
\hline$\Delta$ & $0.0(-1.0-0.0)$ & $0.0(0.0-0.0)$ & NS \\
\hline \multicolumn{4}{|c|}{ Second epoch (6-10 min) } \\
\hline Baseline & $0.0(0.0-1.0)$ & $0.0(0.0-1.0)$ & NS \\
\hline Follow-up review ${ }^{\mathrm{c}}$ & $0.0(0.0-0.0)$ & $0.0(0.0-0.3)$ & NS \\
\hline$\Delta$ & $0.0(-1.0-0.0)$ & $0.0(-1.0-0.3)$ & NS \\
\hline \multicolumn{4}{|c|}{ Third epoch (11-15 min) } \\
\hline Baseline & $0.0(0.0-5.0)$ & $1.0(0.0-2.5)$ & NS \\
\hline Follow-up review ${ }^{\mathrm{c}}$ & $0.0(0.0-0.0)$ & $0.0(0.0-1.0)$ & NS \\
\hline$\Delta$ & $0.0(-4.5-0.0)$ & $0.0(-1.8-0.3)$ & NS \\
\hline \multicolumn{4}{|c|}{ Fourth epoch (16-20 min) } \\
\hline Baseline & $2.0(0.0-5.5)$ & $3.0(0.8-7.8)$ & NS \\
\hline Follow-up review ${ }^{\mathrm{c}}$ & $0.0(0.0-0.5)$ & $0.0(0.0-0.5)$ & NS \\
\hline$\Delta$ & $-2.0(-5.5-0.0)$ & $-1.0(-7.8-0.0)$ & NS \\
\hline \multicolumn{4}{|c|}{ Fifth epoch (21-25 min) } \\
\hline Baseline & $2.0(0.0-4.0)$ & $4.0(0.0-8.5)$ & NS \\
\hline Follow-up review ${ }^{\mathrm{c}}$ & $0.0(0.0-1.0)$ & $0.0(0.0-2.5)$ & NS \\
\hline$\Delta$ & $-1.0(-3.5-0.0)$ & $-2.0(-8.3-0.0)^{*}$ & NS \\
\hline \multicolumn{4}{|c|}{ Slope coefficient of time course SDLP } \\
\hline Baseline & $0.20(0.06-0.60)$ & $0.63(0.04-0.90)$ & NS \\
\hline Follow-up review ${ }^{\mathrm{c}}$ & $0.05(-0.06-0.30)$ & $0.14(-0.22-0.28)$ & NS \\
\hline$\Delta$ & $-0.01(-0.55-0.13)$ & $-0.15(-1.20-0.23)$ & NS \\
\hline
\end{tabular}

$C P A P$ continuous positive airway pressure; $L O A$ lapses of attention; $N S$ not significant; $O A$ oral appliance; $S D L P$ standard deviation of the lateral position

${ }^{a}$ Plus-minus values are means \pm standard deviations, values with additives in parenthesis are medians with interquartile ranges.

${ }^{\mathrm{b}}$ Significance for the difference in baseline, follow-up, or $\Delta$ outcomes between treatment groups.

${ }^{\mathrm{c}}$ The median treatment period from baseline until final follow-up review was 81 (interquartile range $72-93$ ) days in the OA group and 79 (interquartile range 63-102) days in the CPAP group $(p>0.05)$.

$* p=0.04$ for difference between baseline and follow-up outcomes within treatment group

$*^{*} p=0.03$ for difference between baseline and follow-up outcomes within treatment group

Therefore, based on the definition criteria employed in the present study [22], the chance of having included patients with OSAHS in the control group is unlikely. A second limitation refers to a possible "learning effect" on the driving simulator when evaluating patients at the final follow-up review. Previous studies have failed to demonstrate profound learning effects in simulated driving performance in OSAHS patients [7, 30]. Our driving simulator test could easily be performed by all subjects because the difficulty level was low and subjects were allowed to master the task during a practice session. If a learning effect would have been present, it would therefore have had a limited effect on the performance during the second driving test. In addition, any residual learning effect was controlled for between the two treatments through the use of a parallel study design. Thirdly, changes in simulator performance may (at least in part) result from a placebo effect. In addition, simulator performance may be influenced by volition and motivation, which may add further noise to the observed improvements in simulator performance. Trials comparing placebos with therapeutic CPAP, however, have failed to demonstrate strong placebo effects with respect to simulated driving performance [14]. A fourth limitation may be the fact that patient's reported treatment usage was not objectified in the present study. Whereas CPAP usage can be monitored covertly with a mechanism built into the device, oral-appliance usage cannot be assessed covertly in any reliable way. To eliminate bias, we ensured that treatment usage was assessed in the same manner by basing the assessments on self-reports in both groups. Questionnaire analysis indicated adequate treatment usage in both groups. How- 
ever, self-reports usually overestimate the use of treatment by $1 \mathrm{~h} /$ night [31]. When we adjusted for this factor, adherence to treatment was still adequate [32].

From the present investigation, we conclude that OSAHS patients perform worse on a monotonous simulated driving test when compared with control subjects. When evaluating the effects of treatment, adequate OSAHS management with either OA or CPAP therapy usually resulted in substantial improvements of simulated driving. Although our findings suggest a more pronounced effect of CPAP on simulated driving performance, this phenomenon is largely explained by the relatively small study groups and large inter-group variance. Conclusions beyond both treatments improving simulated driving performance are therefore not justified. Studies with larger sample sizes that use a placebo group are warranted to elucidate the specific differences in effects of OA and CPAP therapy on simulated driving performance.

Acknowledgement The authors wish to thank Mr. Bas K. Uildriks for his efforts in the graphics layout of the manuscript. The authors also wish to thank dental laboratory Goedegebuure (Ede, The Netherlands) for their assistance and expertise in manufacturing the oral appliances. The present paper was written in partial fulfilment of the requirements for a Ph.D. degree. Financial support for this M.D.clinical research traineeship was granted by The Netherlands Organisation for Health Research and Development (The Hague, The Netherlands).

\section{References}

1. Malhotra A, White DP (2002) Obstructive sleep apnoea. Lancet 360:237-245

2. George CF (2003) Driving simulators in clinical practice. Sleep Med Rev 7:311-320

3. George CF (2004) Sleep 5: driving and automobile crashes in patients with obstructive sleep apnoea/hypopnoea syndrome. Thorax 59:804-807

4. Cassel W, Ploch T, Becker C, Dugnus D, Peter JH, von Wichert P (1996) Risk of traffic accidents in patients with sleepdisordered breathing: reduction with nasal CPAP. Eur Respir J 9:2606-2611

5. Teran-Santos J, Jimenez-Gomez A, Cordero-Guevara J (1999) The association between sleep apnea and the risk of traffic accidents. N Engl J Med 340:847-851

6. Haraldsson PO, Carenfelt C, Lysdahl M, Tornros J (1995) Longterm effect of uvulopalatopharyngoplasty on driving performance. Arch Otolaryngol Head Neck Surg 121:90-94

7. George CF, Boudreau AC, Smiley A (1996) Simulated driving performance in patients with obstructive sleep apnea. Am J Respir Crit Care Med 154:175-181

8. Findley LJ, Suratt PM, Dinges DF (1999) Time-on-task decrements in "steer clear" performance of patients with sleep apnea and narcolepsy. Sleep 22:804-809

9. Juniper M, Hack MA, George CF, Davies RJ, Stradling JR (2000) Steering simulation performance in patients with obstructive sleep apnoea and matched control subjects. Eur Respir J 15:590-595
10. Turkington PM, Sircar M, Allgar V, Elliott MW (2001) Relationship between obstructive sleep apnoea, driving simulator performance, and risk of road traffic accidents. Thorax 56:800-805

11. Brouwer WH (2002) Attention and driving, a cognitive neuropsychological approach. In: Zimmermann P, Leclercq M (eds) Applied neurospsychology of attention. Psychology Press, Hove, pp 223-248

12. George CF (2001) Reduction in motor vehicle collisions following treatment of sleep apnoea with nasal CPAP. Thorax 56:508-512

13. George CF, Boudreau AC, Smiley A (1997) Effects of nasal CPAP on simulated driving performance in patients with obstructive sleep apnoea. Thorax 52:648-653

14. Hack M, Davies RJ, Mullins R, Choi SJ, Ramdassingh-Dow S, Jenkinson C, Stradling JR (2000) Randomised prospective parallel trial of therapeutic versus subtherapeutic nasal continuous positive airway pressure on simulated steering performance in patients with obstructive sleep apnoea. Thorax 55:224-231

15. Orth M, Duchna H-W, Leidag M, Widdig W, Rasche K, Bauer TT, Walther JW, de Zeeuw J, Malin JP, Schultze-Werninghaus G, Kotterba S (2005) Driving simulator and neuropsychological testing in OSAS before and under CPAP therapy. Eur Respir J 26:898-903

16. Hoekema A, Stegenga B, de Bont LGM (2004) Efficacy and comorbidity of oral appliances in the treatment of obstructive sleep apnea-hypopnea: a systematic review. Crit Rev Oral Biol Med 15:137-155

17. Ferguson KA, Cartwright R, Rogers R, Schmidt-Nowara W (2006) Oral appliances for snoring and obstructive sleep apnea: a review. Sleep 29:244-262

18. Altman DG (1991) Designing research. In: Altman DG (ed) Practical statistics for medical research. CRC Press, Boca Raton, Florida, pp 74-106

19. Johns MW (1991) A new method for measuring daytime sleepiness: the Epworth sleepiness scale. Sleep 14:540-545

20. Pancer J, Al-Faifi S, Al-Faifi M, Hoffstein V (1999) Evaluation of variable mandibular advancement appliance for treatment of snoring and sleep apnea. Chest 116:1511-1518

21. Hoekema A, Stegenga B, van der Aa JG, Meinesz AF, van der Hoeven JH, Wijkstra PJ (2006) Nap-titration; an effective alternative for continuous positive airway pressure titration. Respir Med 100:705-713

22. (1999) Sleep-related breathing disorders in adults: recommendations for syndrome definition and measurement techniques in clinical research. The report of an American Academy of Sleep Medicine Task Force. Sleep 22:667-689

23. (1992) EEG arousals: scoring rules and examples: a preliminary report from the Sleep Disorders Atlas Task Force of the American Sleep Disorders Association. Sleep 15:173-184

24. Rechtschaffen A, Kales AA (1968) Manual of standardized terminology, techniques and scoring system for the sleep stages of human subjects. US Government Printing Office, Washington, DC: NIH Publication No. 204:1-1-0068

25. (1993) Recording and scoring leg movements. The Atlas Task Force. Sleep 16:748-759

26. van Zomeren AH, Brouwer WH, Rothengatter JA, Snoek JW (1988) Fitness to drive a car after recovery from severe head injury. Arch Phys Med Rehabil 69:90-96

27. Brouwer WH, Ponds RW, van Wolffelaar PC, van Zomeren AH (1989) Divided attention 5 to 10 years after severe closed head injury. Cortex 5:219-230

28. Withaar FK, Brouwer WH (2003) Divided attention after closed head injury. Z Neuropsychol 14:203-211

29. Baulk SD, Reyner LA, Horne JA (2001) Driver sleepinessevaluation of reaction time measurement as a secondary task. Sleep 24:695-698 
30. Turkington PM, Sircar M, Saralaya D, Elliott MW (2004) Time course of changes in driving simulator performance with and without treatment in patients with sleep apnoea hypopnoea syndrome. Thorax 59:56-59

31. Kribbs NB, Pack AI, Kline LR, Getsy JE, Schuett JS, Henry JN, Maislin G, Dinges DF (1993) Objective measurement of patterns of nasal CPAP use by patients with obstructive sleep apnea. Am Rev Respir Dis 147:887-895

32. Haniffa M, Lasserson TJ, Smith I (2004) Interventions to improve compliance with continuous positive airway pressure for obstructive sleep apnoea. Cochrane Database Syst Rev 4: CD003531 